\title{
In-Silico Analysis of nsSNPs Associated with CYP11B2 Gene
}

$4 \quad{ }^{1}$ Department of Computer Science, Virtual University of Pakistan, Lahore 54000, Pakistan

$5{ }^{2}$ Department of Bioinformatics and Computational Biology, Virtual University of Pakistan,

6 Lahore 54000, Pakistan

$7{ }^{3}$ Department of Computer Science, COMSATS University Islamabad (Lahore Campus) 54000,

8 Pakistan

9 4Institute of Pharmaceutical Sciences, University of Veterinary and Animal Sciences (UVAS),

10 Lahore 54000, Pakistan

$11{ }^{5}$ Department of Genetics, Virtual University of Pakistan, Lahore 54000, Pakistan

$12{ }^{6}$ Interdisciplinary Research Centre in Biomedical Materials, COMSATS University Islamabad,

13 Lahore Campus, Lahore 54000, Pakistan

14 Corresponding Authors:

15 1- * Dr. Muhammad Tariq Pervez

Contact \# +923033000543

17 E-mail: m.tariq@vu.edu.pk 


\section{Abstract} synthase enzyme and thereby has an essential role to balance salt and mineral level in the body. A

25 lead to many diseases including hypertension and cardiac diseases. To control the excess

26 production of this aldosterone an inhibitor "Fadrozole" is being used which is associated with an

27 active site cavity of CYP11B2. This study has been divided into two parts. In the first part, the

28 four computational tools (SIFT, Polyphen-2, I-Mutant, ConSurf) were used to identify 29

29 deleterious SNPs out of 1600 CYP11B2 SNPs. In the second part, five residues (R448G, R141P, 30 W260R, F130S, and F445S) were identified in the active site cavity (out of 29 deleterious 31 CYP11B2 SNPs) at the distance of $5 \mathrm{~A}^{\circ}$. Binding free energy calculation as well as Dynamics

32 simulation techniques were applied to determine the effect of these mutations on the CYP11B2-

33 Fadrozole compound. The results showed that Fadrozole binding with CYP11B2 became stronger

34 which proved the efficiency of this drug inhibitor with these highly damaging mutations. Our study 35 will be useful for selecting the high priority CYP11B2 mutations, which could be further, 36 investigated in this gene-associated study, for better understanding of the structural and functional 37 aspects of the observed (CYP11B2) protein. 


\section{Introduction}

Human genetic variations are critical in medical and functional perspectives. SNPs (Single

41 Nucleotide Polymorphism) belongs to most abundant classes of genetic variations in the human

42 genome. These contributes in many complicated central nervous system phenotypes, including

43 drug response, susceptibility to neuro-physiological as well as psychiatric disorders. These mutations are highly abundant with a frequency of 1 to 4 out of every 1,00 bases in the human genome [1]]. SNPs are of different types, mostly they are neutral but some of them are functional that can cause an amino acid modification which ultimately can affect protein structure and

47 function, these are known as missense SNPs / nsSNPs (non-synonymous SNP).

48 Scientists have been trying to identify the functional SNPs of human genes for different diseases [2]]. Although lab experiments can accurately identify the genetic role of an SNP [3] but it is not practically possible to test a large number of genetic variants in lab even for a single gene. For

51 example, a single gene like CYP11B2 has more than 1600 SNPs (at the time writing article) which

52 raises a need to prioritize SNPs to minimize this big number to a practical number for lab 53 experiments [4]. This necessity leads researchers to computational study of the genetic variants to 54 predict disease-associated amino acid mutations. The computational tools are fast and reliable in 55 their predictions with accuracy of $80-85 \%$ [5].

56 CYP11B2 is located over the upper layer of the kidney at position 8q24.3 [5]]. It produces

57 aldosterone synthase enzyme and thereby has an essential role to balance salt and mineral level in 58 the body. Any SNP in this gene can deregulate the production of aldosterone hormone in the body 59 which may lead to many diseases including hypertension and cardio vascular disease. [6]]. Brand 60 E, et. Al., found a positive association of CYP11B2-344T allele with essential hypertension [8-9]. 
61 During a study, 175 cardiac patients of European Continental Ancestry Population were

62 diagonosed and it was identified that $\mathrm{C}$ allele $(\mathrm{CT}, \mathrm{CC})$ at $-344 \mathrm{~T} / \mathrm{C}$ SNP position in "aldosterone

63 synthase" gene does not considerably effect clinical prognosis of cardiac heart-failure [7].

64 Similarly the excess of aldosterone has been reported for hypertension [8] which is one of the most

65 prevalent diseases these days around the world and affects more than 1 billion people

66 worldwide[12]. Regular hypertension control medicines work fine but still a large number of

67 patients don't get benefit of them and their blood pressure remains high even after treatment [13].

68 One of the possible solutions used by scientists is the use of an inhibitor (Fadrozole) to limit the

69 biosynthesis of aldosterone hormone [14].

70 Jia et.al [9] studied CYP11B2 gene for 52 SNPs and found four SNPs as deleterious. The novelty 71 of this study was that we filtered out 29 deleterious SNPs from a large number of non-risk alleles

72 and studied the effect of Fadrozole drug over some pathogenic SNPs. There are more than 1600

73 identified SNPs in CYP11B2 gene but only one mutation (-344T/C rs1799998) got primary focus

74 for disease association studies. In this study, we performed computational analysis of CYP11B2

75 gene to identify the pathogenic mutations. The second objective of this study was to identify the

76 effect of missense mutations over receptor-ligand (CYP11B2-Fadrozole) binding; whether these

77 mutations strengthen or weaken the inhibitor affinity with CYP11B2. Fadrozole is an inhibitor

78 used to limit the excess production of aldosterone from CYP11B2 to control diseases e.g.

79 cardiovascular and hypertension. Aldosterone synthase responsibility is to biosynthesize the main

80 mineralocorticoid aldosterone in the adrenal cortex.

81 A computational study on the same gene was performed in 2014 [15] but we monitored four times

82 increase in SNPs (from 358 to >1600: in 2017 when we thought to study this gene) which

83 motivated us to re-investigate nsSNPs associated with CYP11B2 gene. Our study will help 
bioRxiv preprint doi: https://doi.org/10.1101/602615; this version posted April 8, 2019. The copyright holder for this preprint (which was not certified by peer review) is the author/funder, who has granted bioRxiv a license to display the preprint in perpetuity. It is made available under aCC-BY 4.0 International license.

84 biologists to further understand the CYP11B2 gene association with human diseases, and for future 85 experiments.

86 


\section{2. Materials and Methods}

88

89

90

91

92

93

94

95

96

97

98

99

100

101

102

103

104

105

106

\subsection{Dataset collection}

The SNP information i.e. SNPID, Gene Position, mRNA position, Protein Position, Residue Change, mRNA (accession number: NM_000498.3), Protein Sequence (NCBI Accession number: NP_000489.3) of the human CYP11B2 gene were obtained from NCBI (National Center for Biotechnology Information) database of SNPs (dbSNP (http://www.ncbi.nlm.nih.gov/snp/) [10]. The crystal structure of CYP11B2 protein in complex with inhibitor Fadrozole (PDB ID: 4FDH) were obtained from RCSB protein Data Bank (www.rcsb.org). In this study, these (ligands binding) regions were used as active sites for docking. Figure 1 shows the workflow for in-silico analysis of functional SNPs in the human CYP11B2 gene.

\section{Figure 1 shows the workflow, tools, and databases used for in-silico analysis of functional SNPs in the human CYP11B2.}

\subsection{Bioinformatics Tools used to identify deleterious SNPs}

In order to evaluate potential impact of the selected missense SNPs, we utilized four prediction tools and filtered all missense SNPs that were classified as deleterious by all of them. These computational tools include SIFT, I-Mutant, Consurf and Polyphen-2.

SIFT (Sorting Intolerant From Tolerant) is a sequence homology based bioinformatics tool which can be accessed at (http://sift.bii.a-star.edu.sg/index.html) [11]. This tool was used to predict 
107 whether the protein will tolerate the newly introduced amino acid caused by SNP mutation or it 108 will be proved as a damaging mutation [16]. Genome tool "Sift non-synonymous single nucleotide 109 variant (human build 37)" was used in our study. The SIFT input query string includes 110 Chromosome number (8 for CYP11B2), Chromosome position, Orientation and allele 111 respectively. SIFT outputs tolerance index score (Score), SNP mutation of index score $<0.05$ are 112 considered as deleterious. A very low tolerance index indicates that the particular mutation has a 113 more functional impact [12]].

114 Polyphen-2 is a protein sequence and structure based method. This tool was used to foresee the 115 amino acid substitution impact on the structure and function of human protein. This can be 116 accessed at (http://genetics.bwh.harvard.edu/pph2/)[13]. Polyphen-2 query was performed by 117 submitting SNP Identifier/Accession \#, protein position and Substitutions / Residue changes. This 118 computational tool output a large heat-map color bar that range from 0 to 1 to show damaging 119 probability of an SNP. A high score shows the higher chances of a mutation as damaging.

120 The protein stability over single mutation was measured using I-Mutant Suite which can be 121 accessed at (http://gpcr2.biocomp.unibo.it/cgi/predictors/I-Mutant3.0/I-Mutant3.0.cgi) [14]. Its 122 input includes protein position, new residue information and protein sequence (obtained from 123 Uniprot against entry P19099). The output was used to classify Predicted Free Energy 124 change value $(\mathrm{DDG})$ of protein. $\mathrm{DDG}<-0.5\left(\mathrm{kcal} \mathrm{mol}^{-1}\right)$ shows huge decrease in protein-stability 125 on SNP mutation which is critical.

126 Highly conserved regions in proteins/nucleic-acid-sequence often indicate structural and/or 127 functional importance [18]. Consurf web server which can be accessed at 
128 (http://consurf.tau.ac.il/2016/)[15]. It calculates the evolutionary conserved amino acid positions

129 in proteins using Empirical Bayesian inference (protein structure and sequence respectively).

130 Amino acid analysis was performed for PDBID (4FDH) and identifier ' $\mathrm{A}$ '. The conservation

131 scores were calculated among protein and its homology whose color scale ranges from 1 to 9 ,

132 where the scores in the range 7 to 9 indicate conserved region, probability in ascending order.

\subsection{Molecular Docking}

134 The co-crystallized structure for CYP11B2 bonded to Fadrozole (CYP11B2-Fadrozole complex)

135 was retrieved from the Protein Data Bank (PDBID: 4FDH). The initial conformation of Fadrozole

136 in 4FDH has been used as starting point for molecular docking. Prior to Dock the inhibitor into the

137 active site, the receptor-protein structure was prepared (with the help of structure preparation

138 tools) to validate the chemical accuracy in biopolymer module of SYBYL-X 1.3[16]. For that

139 purpose, at first step missing hydrogen atoms added, then all crystallographic waters molecules

140 were removed, then atom-types were assigned, next, atomic charges were applied according to

141 AMBER 7 FF99 force-field. The Power algorithm was used to minimize the energy of each

142 complex, for 1000 cycles at convergence-gradient of $0.5 \mathrm{kcal}\left(\mathrm{mol} \mathrm{A}^{\circ}\right)$. The backbone atoms were

143 kept fixed during structure optimization.

144 Five different in-silico mutants of CYP11B2 (R448G, F445S, W260R, R141P and F130S) were

145 generated by replacing Arg448, Phe445, Trp260, Arg141 and Phe130 with Glycine448, Serine445,

146 Arginine260, Proline141 and Serine130 amino acids respectively. All mutations were induced

147 using SYBYL-X 1.3 (Biopolymer module)[16]. Prior to molecular docking, the generated mutant

148 models of CYP11B2 were energy-minimized and MD-simulated at least for $10 \mathrm{ns,} \mathrm{which} \mathrm{have}$ 
149 been discussed in a later section. For molecular docking, the active structural conformation of

150 Fadrozole was extracted from its complex 4FDH.

151 Finally the active sites of these five mutants of CYP11B2 were docked by the ligand using the

152 same protocol as reported in our previous publications [10-12]. Twenty best-docked conformations

153 were retrieved for each ligand-mutant complex system. The putative ligands poses were ranked

154 according to Hammerhead scoring function (C-score) [4]. To examine the effect of the point 155 CYP11B2 mutation over receptor-ligand interaction, all ligand protein complexes were visualized 156 and critically investigated.

\section{$157 \quad 2.4$ Molecular dynamics Simulation}

158 For all five generated structures of the complexes (Fadrozole-CYP11B2, Fadrozole- R448G, 159 Fadrozole-F445S, Fadrozole-W260R, Fadrozole- R141P and Fadrozole-F130S) was further 160 stabilized by performing MD simulations. These simulations were performed with the SANDER 161 module in AMBER12 software package in a solvation system. LEaP program embedded in 162 AMBER12 was used to add missing hydrogen atoms of protein. The complex was immersed into 163 an octahedron box of TIP3P water molecules to achieve water molecules up-to minimum $10^{\circ} \mathrm{A}$ 164 distance between protein and edge of box. Extra water molecules were replaced by $\mathrm{Cl}^{-} / \mathrm{Na}^{+}$ 165 counter-ions by using LEaP protocol.

166 AMBER ff99SB force field, for protein, and General AMBER force field (GAFF together with 167 RESP charges), for ligand, was used to assign force field parameters for each protein-ligand 168 complex. 
169 Each protein-ligand complex of the mutant and wild type was then subjected to production

170 simulations run of $50 \mathrm{~ns}$ by keeping protocol and parameters same as those reported in our previous

171 publications [10-12]. All these analyses were performed using ANAL, CARNAL and

172 PTRAJ modules of 'AMBER12' software.

173

\subsection{MM/PB(GB)SA Based Free energy calculation}

$174 \mathrm{MM} / \mathrm{PB}(\mathrm{GB}) \mathrm{SA}[13]$, a molecular-mechanics based scoring method, was used to compare the

175 binding free energies of Fadrozole-R448G, Fadrozole-F445S, Fadrozole-W260R, Fadrozole-

176 R141P and Fadrozole-F130S mutant complexes to wild type Fadrozole-CYP11B2. The pairwise

177 nature of the GB methodology allows to produce insightful interaction and desolation components

178 by decomposing free-energies. All MM/PB(GB)SA calculations [13] were thoroughly performed

179 in the AMBER16 software package, while keeping protocol and parameters same as provided in 180 our previous publications $[10,12]$.

181

182 3. Results

\section{$183 \quad 3.1 \quad$ Identification of Deleterious nsSNPS}

185 we started work; now there is more than 600 nsSNPs) was performed (Table S1: Given in 186 Supporting Information Table S1) using SIFT, I-Mutant, Polyphen-2 and computational 187 bioinformatics tools. SIFT was run for 275 SNP (Table S2) and it predicted $98(35.63 \%)$ nsSNPs 
as damaging, while 162 were of benign effect. Polyphen-2 was run for 275 SNP out of which 128 SNPs (46.54\%) were predicted as damaging (Table S3) while 147 mutations were resulted as benign; I-Mutant (structure based tool) was run for total 186 nsSNPs $(67.63 \%)$ which resulted that 117 nsSNPs $(62.9 \%)$ show a DDG $<-0.5$ hence they are largely unstable (Table S4).

The combined results of these three computational tools screened down the CYP11B2 nsSNPs from 275 to $57(20.72 \%)$. Thus 57 mutations (Given in supporting information S5 Table) were predicted by all these three tools that had higher probability of damaging protein structure and cause disease. Figure 2 shows the distribution of benign and deleterious and nsSNPs found using I-Mutant suite, Polyphen and SIFT.

Fig 2. Distribution of deleterious and benign nsSNPs by bioinformatics tools Polyphen-2, SIFT, and I-Mutant Suit.

\subsection{Evolutionary Conservation analysis of nsSNPs}

It is a known fact that mutation in highly conserved protein regions keeps more chances to cause disease against any mutation. The 4FDH.pdb protein structure was analyzed using Consurf to find the conserved regions. ConSurf identified 174 conserved amino acid positions of 4FDH (Table S6). When compared with 57 nsSNP (predicted as damaging by Polyphen, I-Mutant and SIFT), only 29 nsSNPs were found in conserved area (Table 1). 


\begin{tabular}{|c|c|c|c|c|}
\hline Sr. & SNIP ID & Gene Position & mRNA Position & Protein position \\
\hline 1 & rs562670189 & 9459 & 1024 & 341 \\
\hline 2 & rs5315 & 10124 & 1211 & 403 \\
\hline 3 & rs5317 & 10811 & 1462 & 487 \\
\hline 4 & rs369953763 & 8077 & 740 & 246 \\
\hline 5 & rs370011030 & 9460 & 1025 & 341 \\
\hline 6 & rs370056488 & 5127 & 127 & 42 \\
\hline 7 & rs370286183 & 9438 & 1003 & 334 \\
\hline 8 & rs374764229 & 8572 & 949 & 316 \\
\hline 9 & rs375063578 & 9406 & 971 & 323 \\
\hline 10 & rs376248857 & 10258 & 1345 & 448 \\
\hline 11 & rs376776280 & 9465 & 1030 & 343 \\
\hline 12 & rs544064549 & 7796 & 596 & 198 \\
\hline 13 & rs551657406 & 10150 & 1237 & 412 \\
\hline 14 & rs567226780 & 5712 & 325 & 108 \\
\hline 15 & rs 745436748 & 5757 & 370 & 123 \\
\hline 16 & rs746840967 & 10139 & 1226 & 408 \\
\hline 17 & rs 749800670 & 7963 & 626 & 208 \\
\hline
\end{tabular}




\begin{tabular}{|c|c|c|c|c|}
\hline 18 & rs750090886 & 9433 & 998 & 332 \\
\hline 19 & rs753349034 & 10250 & 1337 & 445 \\
\hline 20 & rs 755947763 & 9559 & 1124 & 374 \\
\hline 21 & rs 762371852 & 7790 & 590 & 196 \\
\hline 22 & rs 769090647 & 9417 & 982 & 327 \\
\hline 23 & rs 771766541 & 10196 & 1283 & 427 \\
\hline 24 & rs772456092 & 10027 & 1193 & 397 \\
\hline 25 & rs773983994 & 8118 & 781 & 260 \\
\hline 26 & rs 776605380 & 7625 & 425 & 141 \\
\hline 27 & rs 780325282 & 10306 & 1393 & 464 \\
\hline 28 & rs1025885941 & 10790 & 1441 & 480 \\
\hline 29 & rs1029475315 & 9445 & 1010 & 336 \\
\hline
\end{tabular}

\section{$211 \quad 3.3 \quad$ Molecular Docking}

212 The docking identified 18 residues at the distance of $5 \dot{\mathrm{A}}$ from the active site of CYP11B2-

213 Fadrozole compound, these were R110,W116,R120,F130,W137,R141,F231,W260R, A313, S315,

214 T318, L319, L373, A384,G445, R448 and C450. The next step was to filter out only those residues

215 out of 18 residues which were identified as damaging (Table 1), hence only five residues were

216 subjected for further analysis. R448G and R141P reside were found at $3 \dot{A}$ from drug fadrozole 
217 in CYP11B2-fadrozole complex (Fig 3A) while G445, W260R and F130S reside at the distance

218 of $5 \dot{\mathrm{A}}$ shown in (Fig 3B).

219

Fig 3. Amino acids at different distances from drug Fadrazole

Table 2: Damaging Conserve Area SNPs near Fadrozole-CYP11B2 compound

\begin{tabular}{|l|c|c|c|}
\hline Sr. & SNIP ID & Amino Acid change & Protein Position \\
\hline 1 & rs376248857 & R448G & 448 \\
\hline 2 & rs753349034 & F445S & 445 \\
\hline 3 & rs773983994 & W260R & 260 \\
\hline 4 & rs776605380 & R141P & 141 \\
\hline 5 & rs965343088 & F130S & 130 \\
\hline
\end{tabular}

224 Docking results revealed that there were no particular changes in binding modes of ligands in

225 mutant and wild-type, however, this was not surprising because the binding pocket was almost the

226 same and only one residue was different. The docking scores revealed that the ligand bonded to

227 the SNPs with higher affinity than wild-type/native; however, to further confirm the docking 228 results molecular dynamic simulations were initiated. 


\subsection{Molecular Dynamic Simulation:}

Although, molecular docking analysis is sufficient to depict ligand-receptor interactions. The

231 docking can be further refined with more reliable molecular dynamic (MD) simulation approach.

232 As docking scores cannot be considered as 100\% reliable to distinguish ligands on their selectivity

233 basis. Moreover, in molecular docking approach physiological conditions are not considered.

234 Thus, post-processing molecular docking results with MD simulation may would be helpful to

235 identify dynamic stability of ligand-protein complex. To clarify the structural consequences due 236 to these five identified mutations, docking results were subjected to Molecular Dynamic (MD) 237 simulation analysis.

Fig.4.The RMSD of the backbone atoms of protein (black), backbone atoms of binding pocket residues around $5^{\circ} \mathrm{A}$ of ligand (red), and the heavy atoms in the ligand (blue) for (A) F130S (B) F445S (C) R141P (D) R448G and (E) W260R bound CYP11B2 in complexes with respect to the initial structures as a function of time 40ns (nanosecond).

To examine the stability of the Fadrozol-CYP11B2 complex stability under the simulation conditions the RMSD (root mean square deviation) of the trajectories was measures from their

245 initial structure. The analysis of the RMSD for the active site residues around $5 \dot{\mathrm{A}}$ of ligand 246 backbone atoms and ligand heavy atoms performed for 40ns are observed.

247 Figure 4 shows that the protein backbone atoms RMSD of Fadrozole inhibitor bound CYP11B2 248 mutant complexes (F130S, F445S, R141P, W260R and R448G) in the simulation fluctuates around 
250

251

252

253

254

255

256

257

258

ns, respectively. These results reveals the stability of the docked complex structure throughout MD simulation, although protein structure shows some fluctuations, while the ligand exhibits smaller conformational changes. The low fluctuation in RMSD plot demonstrate that the results are in accordance with ligand-protein (residues) high affinity that are forming a stable complex. Among five ligand-receptor complexes F445S exhibited lowest atomic fluctuations. Hence, it concludes the ligand stability in the binding pocket such that it did not dissociate from the protein during the simulation. Overall, these analyses for ligand-protein complex stabilization suggest that the simulated docking conformation are correct and can be used to calculate binding free energies.

\subsubsection{Binding free energy analysis:}

The binding affinity of ligand towards CYP11B2 and its mutations were calculated using Molecular Mechanics/Poisson-Boltzmann Surface Area (MM/PBSA and MM/GBSA) methodologies[17]. The binding free energy (BFE) was calculated in terms of solvation energy and entropic contributions over 1000 snapshots for the complex, receptor and ligand respectively, obtained over last 5 nano-seconds of MD trajectories for protein-ligand system. Table 3 and Fig 3 (A-E) summarizes computed BFEs and their enthalpy and entropic contributions for five systems (fadrozole-R448G, fadrozole-R141P, fadrozole-W260R, fadrozole- F130S and fadrozole-F445S).

The Fadrozole inhibitor possesses higher predicted binding energies for F445S $\left(\Delta \mathrm{G}_{\text {pred }}(\mathrm{GB})\right.$ MMGBSA: -19.88 $\mathrm{kcalmoli}^{-1}$; MMPBSA: -28.13 $\mathrm{kcalmol}^{-1}$ ) and indicate more significant difference in $\Delta \mathrm{Gpred}_{(\mathrm{GB} / \mathrm{PB})}$ values for F445S vs R448G, R141P, W260R and F130S. Still it can be seen that the binding energy increases for all five mutants of fadrozol-CYP11B2 than wild type.

Table 3: Binding free energy change due to five mutations in the Fadrozole-CYP11B2 compound. 


\begin{tabular}{|c|c|c|c|c|c|c|}
\hline Protein-inhibitor & $\begin{array}{l}\text { Wild- } \\
\text { Type } \\
\text { (4FDH) }\end{array}$ & 4FDH_R448G & R141P & W260R & F130S & F445S \\
\hline$\Delta \mathbf{E}_{\mathbf{v d W}}$ & -24.98 & -27.35 & -33.18 & -29.59 & -29.12 & -29.58 \\
\hline$\Delta \mathbf{E}_{\text {ele }}$ & -15.39 & -5.36 & -10.34 & -2.16 & -10.50 & -22.77 \\
\hline$\Delta \mathbf{G}_{\text {nonpol, sol }}$ & -3.07 & -3.79 & -4.41 & -3.96 & -3.71 & -3.91 \\
\hline$\Delta \mathbf{G}_{\text {ele, sol (PB) }}$ & 30.89 & 21.95 & 27.78 & 17.84 & 29.82 & 35.56 \\
\hline$\Delta \mathbf{G}_{\text {ele, sol (GB) }}$ & 23.76 & 14.43 & 17.76 & 10.34 & 19.40 & 28.11 \\
\hline 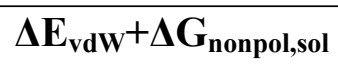 & -28.05 & -31.13 & -37.59 & -33.55 & -32.83 & -33.48 \\
\hline$\Delta \mathbf{E}_{\text {ele }}+\Delta \mathbf{G}_{\text {ele,sol (PB) }}$ & 15.50 & 16.59 & 17.43 & 15.68 & 19.32 & 12.80 \\
\hline$\Delta \mathbf{E}_{\text {ele }}+\Delta \mathbf{G}_{\text {ele,sol (GB) }}$ & 8.37 & 9.07 & 7.42 & 8.18 & 8.90 & 5.35 \\
\hline$\Delta \mathrm{G}_{\text {pred (PB) }}$ & -12.60 & -13.99 & -19.05 & -17.27 & -12.84 & -19.88 \\
\hline$\Delta \mathbf{G}_{\text {pred (GB) }}$ & -19.68 & -22.06 & -30.17 & -25.38 & -23.93 & -28.13 \\
\hline
\end{tabular}

271 In order to identify the driving forces for selective bindings of ligand on inhibitor, total binding

272 free energy was decomposed into independent (binding free energy) components. (Fig 34-E or

273 Table 3) using MMGB(PB)SA methods. 
Fig 5 Comparison between binding free energy terms of Fadrozole

$$
\text { (A) R448G, (B) R141P, (C) W260R, (D) F130S, (E)F445S }
$$

The calculated values of individual binding free energy components for four systems (Fig. 5 A-D) revealed that the disfavor-able electrostatic interactions between protein and ligand $\left(\Delta \mathbf{E}_{\mathbf{e l e})}\right.$ in vacuum were opposed by the favorable electrostatic energy of solvation $\left(\Delta \mathbf{G}_{\text {ele,sol }}\right)$. However, the sum of the electrostatic interaction contributions of $\left(\Delta \mathbf{E}_{\mathbf{e l e}}\right)$ and $\left(\Delta \mathbf{G}_{\text {ele,sol }}\right)$ slightly disfavors the protein-ligand binding. F445S shows the opposite behavior, $\left(\Delta \mathbf{E}_{\text {ele }}\right)$ decreases and $\left(\Delta \mathbf{G}_{\text {ele,sol }}\right)$ increases, and sum of these two favors this binding. The sum of vdW energy $\left(\Delta \mathbf{E}_{\mathbf{v d W}}\right)$ and nonpolar solvation energy ( $\left.\Delta \mathbf{G}_{\text {nonpol,sol }}\right)$ is favorable contribution to inhibitor binding to CYP11B2 for all five mutant systems. The total vdW contribution for fadrozole-CYP11B2 complex was -28.05 kcal.mol ${ }^{-1}$. However, total predicted binding free energy in terms of both $\Delta \mathbf{G}_{\text {pred (PB) }}$ and $\Delta \mathbf{G}_{\text {pred }}$ (GB) Was reduced for all mutants and maximum favorable binding was observed for R141P (-19.05 kcal.mol-1) and F445S (-19.88 kcal.mol) nsSNPs mutants.

\subsubsection{Insight into the binding affinity of Fadrozole compound to CYP11B2:}

For all receptor-ligand complexes, the binding free energies were decomposed on interaction energies of Fadrozol with residues to estimate the role of distinct residue in tight binding of inhibitor to active site of protein. In case of R141, ten amino acids TRP83, ARG87, MET197, PHE198, GLU277, ALA280, GLY281, SER282, ILE449, GLU277 have favorable binding free energy, whereas five amino acids were disfavor-able (shown in Fig 6A-E). For F445S, mutant has

low binding free energy for TRP83, ARG87, GLU277, ALA280, GLY281, PHE97, LEU98, LEU412 which contributed towards strong interaction with Fadrazole inhibitor. 
Analysis of free energy components showed that increase in selectivity was directly associated with reduction of binding free energy of amino acids.

\section{Discussion}

The excess of aldosterone has been reported to cause the hypertension [8], which is one of the most prevalent diseases these days around the world and affected more than 1 billion people worldwide[12]. Regular hypertension control medicines work fine but still a large number of patients don't get benefit of them and their blood pressure remains high even after treatment [13]. One of the possible solutions used by scientists is the use of an inhibitor (Fadrozole) to limit the biosynthesis of aldosterone hormone [14]. The inhibitor binds with CYP11B2 gene at a specific binding cavity. This binding strength between inhibitor and CYP11B2 gene depends on interaction energies of inhibitor with the residues of protein (active cavity site/ nucleotides). Any mutation in these nucleotides at binding sites may affect the binding free energies of receptor-ligand complex and hence can alter inhibitor efficiency. Almost 1600 SNPs of CYP11B2 has been reported till 2018 (https://www.ncbi.nlm.nih.gov/snp). In this study, we performed computational analysis of CYP11B2 gene to identify the pathogenic mutations and the effect of missense mutations over receptor-ligand (CYP11B2-Fadrozole) binding; whether these mutations strengthen or weaken the inhibitor affinity with CYP11B2 [18].

This study prioritizes SNPs with functional significance from an enormous number of non-risk alleles and provides new insights for further genetic association studies. Of 1600 SNPs, we 
320

321

322

323

324

325

326

327

328

329

330

331

332

333

334

335

336

337

338

selected about 275 missense SNPs for our investigations. In the first phase of our research we identified 57 deleterious nsSNPs using Sequence and structure based tools namely SIFT, Polyphen and I-Mutant (Table S7: Supporting Table S7), in second phase we identified evolutionary conserved areas of 4FDH (PDB of CYP11B2) (Table S6) due to the importance of the fact that mutation in evolutionary conserved areas are more liable for disease causing[19] and filter 29 of 57 highly deleterious SNPs that resides in conserved protein areas. In the third phase we add another dimension into our research, and check that if any of these nsSNPs has effect on drug Fadrozole.

Docking and Molecular dynamic simulation studies have been performed to calculate the binding energy of Fadrozole-CYP11B2 complex. The 4FDH protein structure was analyzed in a docking software and it was found that five (R448G, R141P, W260R, F130S, F445S) out of 29 SNPs were located near fadrozole-CYP11B2 biding cavity. To further understand the structural consequences of the five identified mutations, docking results were analyzed by Molecular Dynamic (MD) simulation. The simulations were performed for both wild-type and mutant nsSNPs the results showed that binding energy of complex increased even after these deleterious mutations.

The literature on CYP11B2 SNPs with disease causing probability is scarce. One mutation (344T/C rs1799998) has been reported for disease association studies [20, 21].

\section{Conclusion}

This study identified 29 deleterious CYP11B2 mutations using four computational tools including SIFT, Polyphen-2, I-Mutant, and Consurf. Then the structural analysis was performed using Sybyl and MD simulation to check the performance of Fadrozole over these mutations. The results have effectively shown that the fadrozole drug efficiently blocks to the identified damaging mutations thus is a very effective and potent drug. 


\section{Acknowledgement:}

343 This project does not have any funding.

\section{References}

345 1. Karki R, Pandya D, Elston RC, Ferlini C. Defining "mutation" and "polymorphism" in the era of

346 personal genomics. BMC Med Genomics. 2015;8(37):015-0115

347 2. Shastry BS. SNP alleles in human disease and evolution. J Hum Genet. 2002;47(11):561-6

$348 \quad 3 . \quad$ Chen X, Sullivan PF. Single nucleotide polymorphism genotyping: biochemistry, protocol, cost and

349 throughput. Pharmacogenomics J. 2003;3(2):77-96

350 4. Vaser R, Adusumalli S, Leng SN, Sikic M, Ng PC. SIFT missense predictions for genomes. Nat Protoc.

$351 \quad 2016 ; 11(1): 1-9$

352 5. Taymans SE, Pack S, Pak E, Torpy DJ, Zhuang Z, Stratakis CA. Human CYP11B2 (aldosterone 353 synthase) maps to chromosome 8q24. 3. The Journal of Clinical Endocrinology \& Metabolism. $354 \quad 1998 ; 83(3): 1033-6$

355 6. Nanba K, Vaidya A, Williams GH, Zheng I, Else T, Rainey WE. Age-Related Autonomous 356 Aldosteronism. Circulation. 2017;136(4):347-55

357 7. Feola $M$, Monteverde $M$, Vivenza $D$, Testa $M$, Leto $L$, Astesana $V$, et al. Prognostic Value of 358 Different Allelic Polymorphism of Aldosterone Synthase Receptor in a Congestive Heart Failure European 359 Continental Ancestry Population. Arch Med Res. 2017;48(2):156-61

360 8. Struthers AD, MacDonald TM. Review of aldosterone-and angiotensin II-induced target organ 361 damage and prevention. Cardiovascular research. 2004;61(4):663-70

362 9. Jia M, Yang B, Li Z, Shen H, Song X, Gu W. Computational analysis of functional single nucleotide 363 polymorphisms associated with the CYP11B2 gene. PloS one. 2014;9(8):e104311 
10. Sherry ST, Ward MH, Kholodov M, Baker J, Phan L, Smigielski EM, et al. dbSNP: the NCBI database

365 of genetic variation. Nucleic Acids Res. 2001;29(1):308-11

366 11. Kumar P, Henikoff S, Ng PC. Predicting the effects of coding non-synonymous variants on protein

367 function using the SIFT algorithm. Nature protocols. 2009;4(7):1073

368 12. Ng PC, Henikoff S. Predicting deleterious amino acid substitutions. Genome research.

$369 \quad 2001 ; 11(5): 863-74$

370 13. Adzhubei IA, Schmidt S, Peshkin L, Ramensky VE, Gerasimova A, Bork P, et al. A method and server

371 for predicting damaging missense mutations: Nat Methods. 2010 Apr;7(4):248-9. doi:

$372 \quad 10.1038 / \mathrm{nmeth} 0410-248$.

373 14. Capriotti E, Calabrese R, Casadio R. Predicting the insurgence of human genetic diseases 374 associated to single point protein mutations with support vector machines and evolutionary 375 information2006.

376 15. Glaser F, Pupko T, Paz I, Bell RE, Bechor-Shental D, Martz E, et al. ConSurf: identification of 377 functional regions in proteins by surface-mapping of phylogenetic information. Bioinformatics. $378 \quad 2003 ; 19(1): 163-4$

379 16. SYBYL X. version 1.3. Tripos, LP: St Louis. 2011

380 17. Kollman PA, Massova I, Reyes C, Kuhn B, Huo S, Chong L, et al. Calculating structures and free 381 energies of complex molecules: combining molecular mechanics and continuum models. Accounts of 382 chemical research. 2000;33(12):889-97

383 18. Zhu M, Zhao S. Candidate gene identification approach: progress and challenges. International 384 journal of biological sciences. 2007;3(7):420-7

385 19. Mooney SD, Klein TE. The functional importance of disease-associated mutation. BMC bioinformatics. 2002;3:24-.10.1186/1471-2105-3-24 
387 20. Androulakis E, Tousoulis D, Papageorgiou N, Miliou A, Chatzistamatiou E, Moustakas G, et al.

388 Effects of the C-344T aldosterone synthase gene variant on preclinical vascular alterations in essential

389 hypertension. International journal of cardiology. 2013;168(2):1605-6

390 21. Freel E, Ingram M, Wallace A, White A, Fraser R, Davies E, et al. Effect of variation in CYP11B1 and

391 CYP11B2 on corticosteroid phenotype and hypothalamic-pituitary-adrenal axis activity in hypertensive

392 and normotensive subjects. Clinical endocrinology. 2008;68(5):700-6

393

394 Supporting Information Caption

395 S1 Table: 275 missense SNPs of CYP11B2

396 S2 Table: SIFT predicted Damaging SNPs

397 S3 Table: Polyphen-2 predicted damaging SNPs

398 S4 Table: I-Mutant predicted Damaging SNPs

399 S5 Table: Damaging nsSNPs by SIFT, POLYPHEN, I-Mutant and ConSurf

400 S6 Table: ConSurf predicted Conserved areas in 4FDHstructure of CYP11B2

401 
PDB

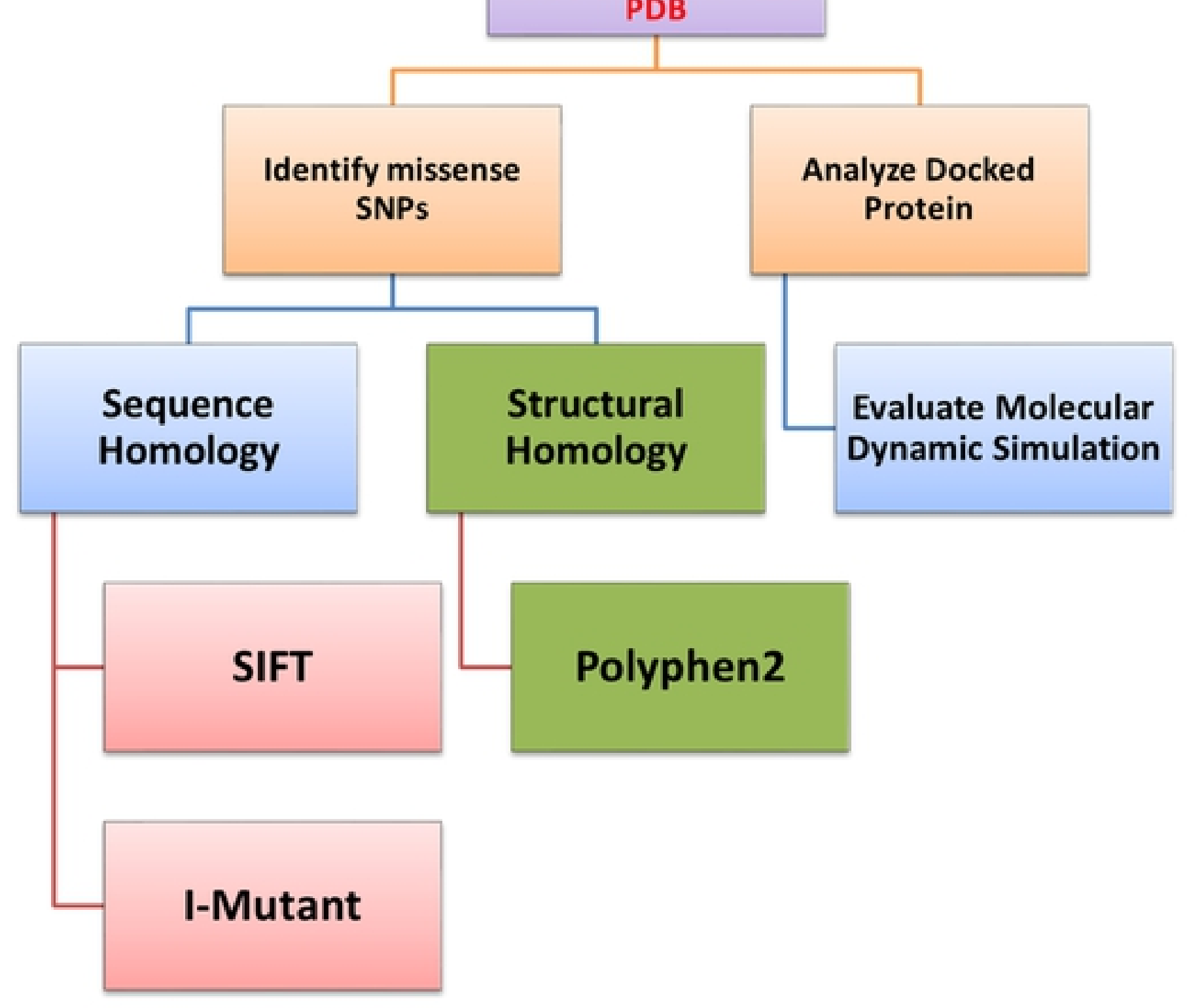

Fig 1 


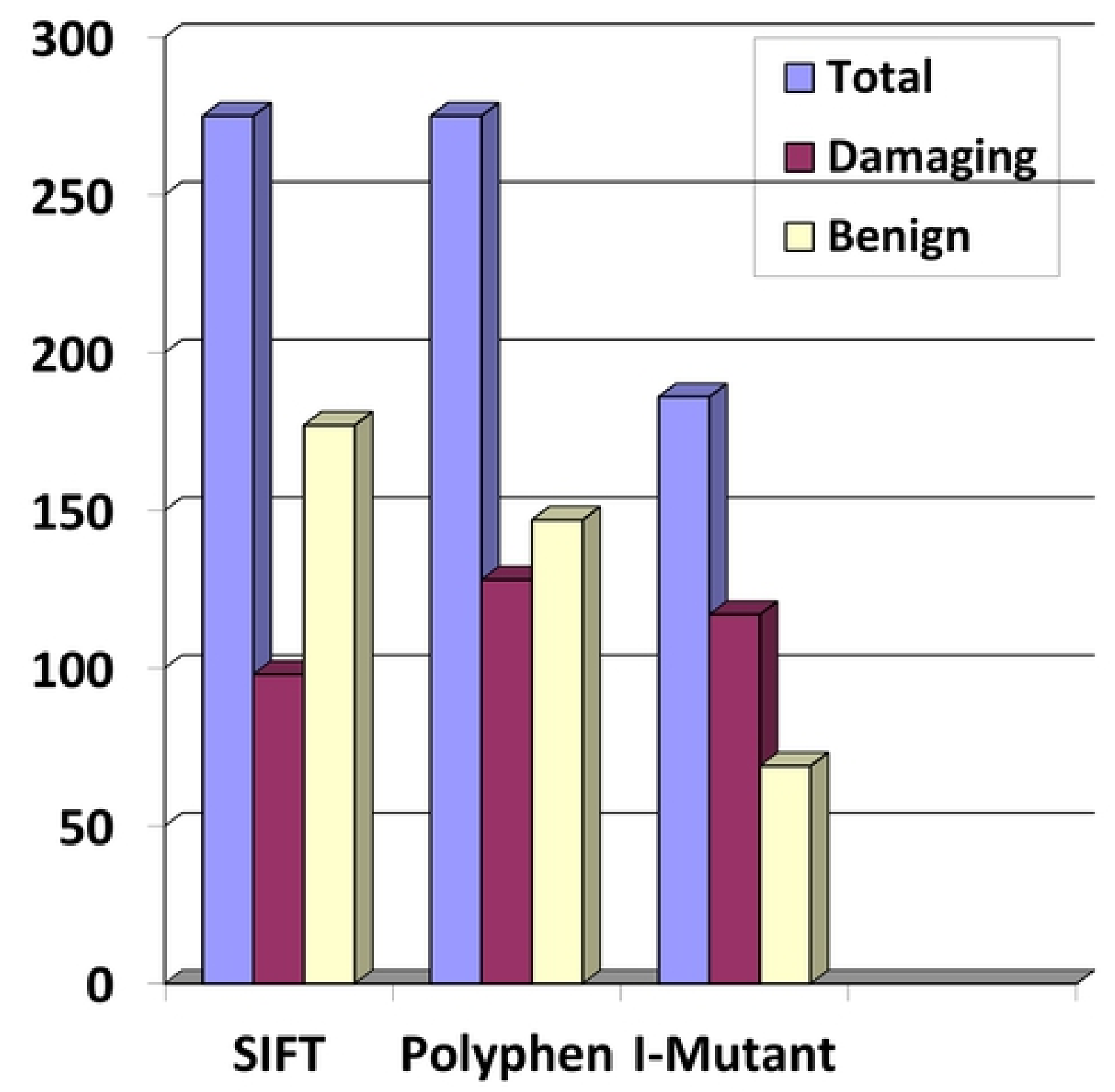

Fig 2 


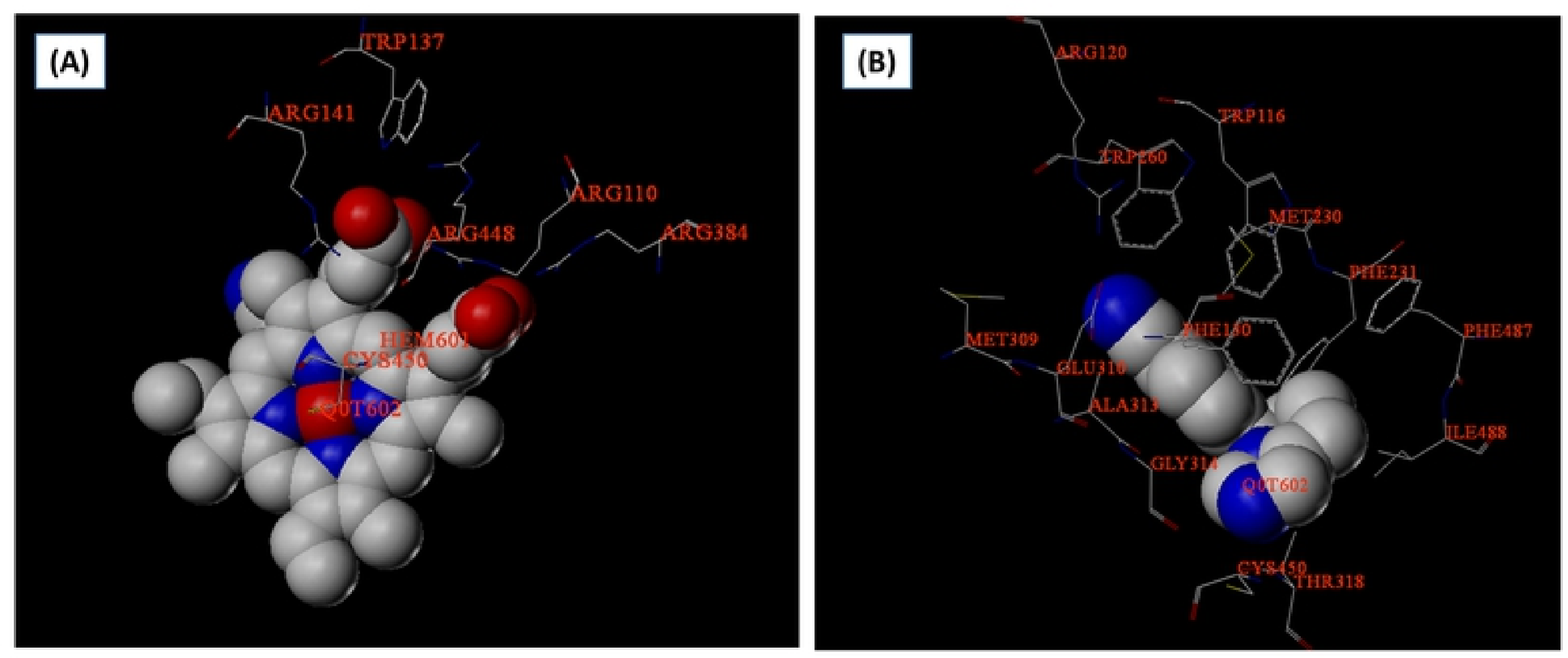

Fig 3 

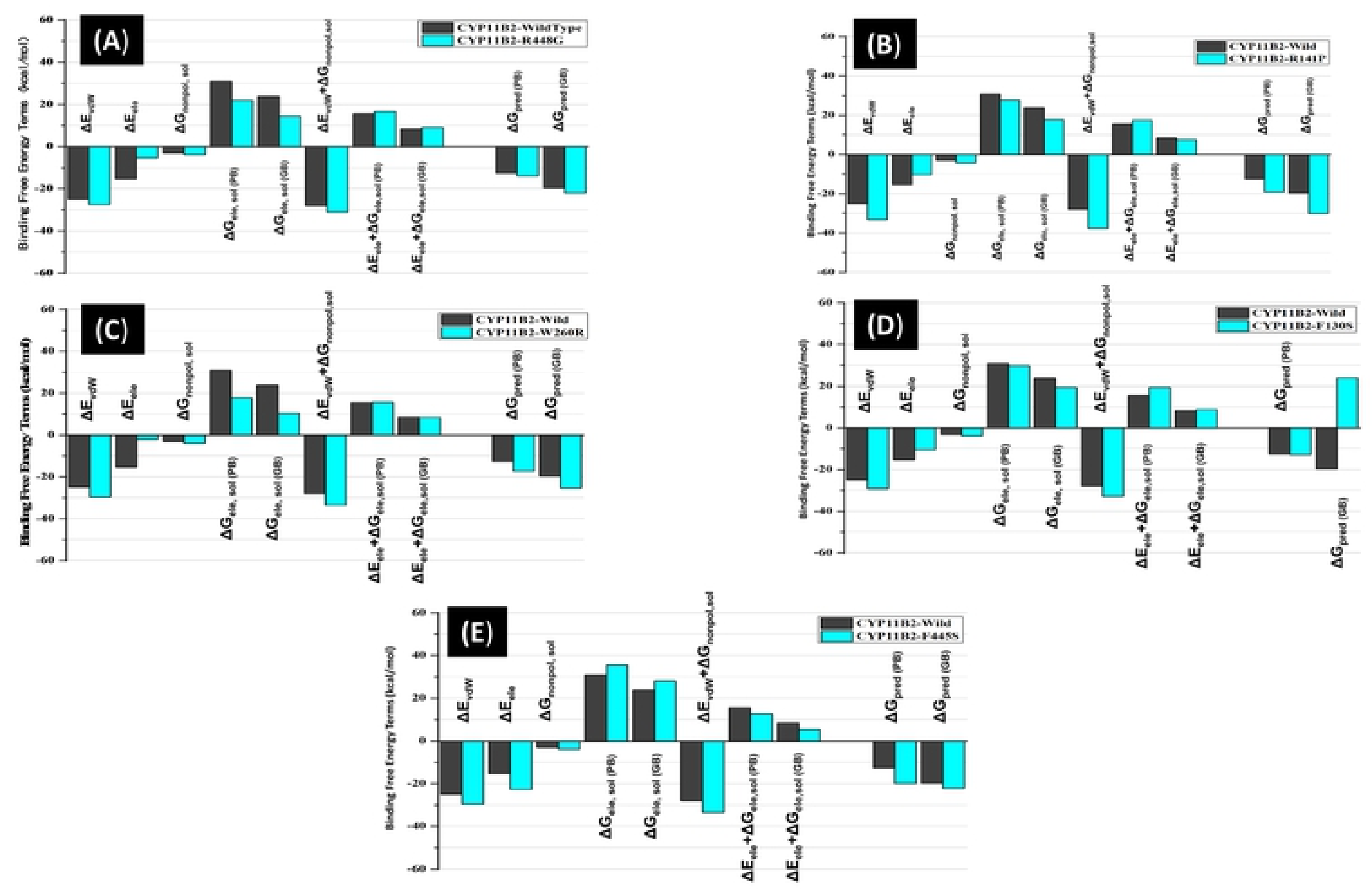

Fig 5 


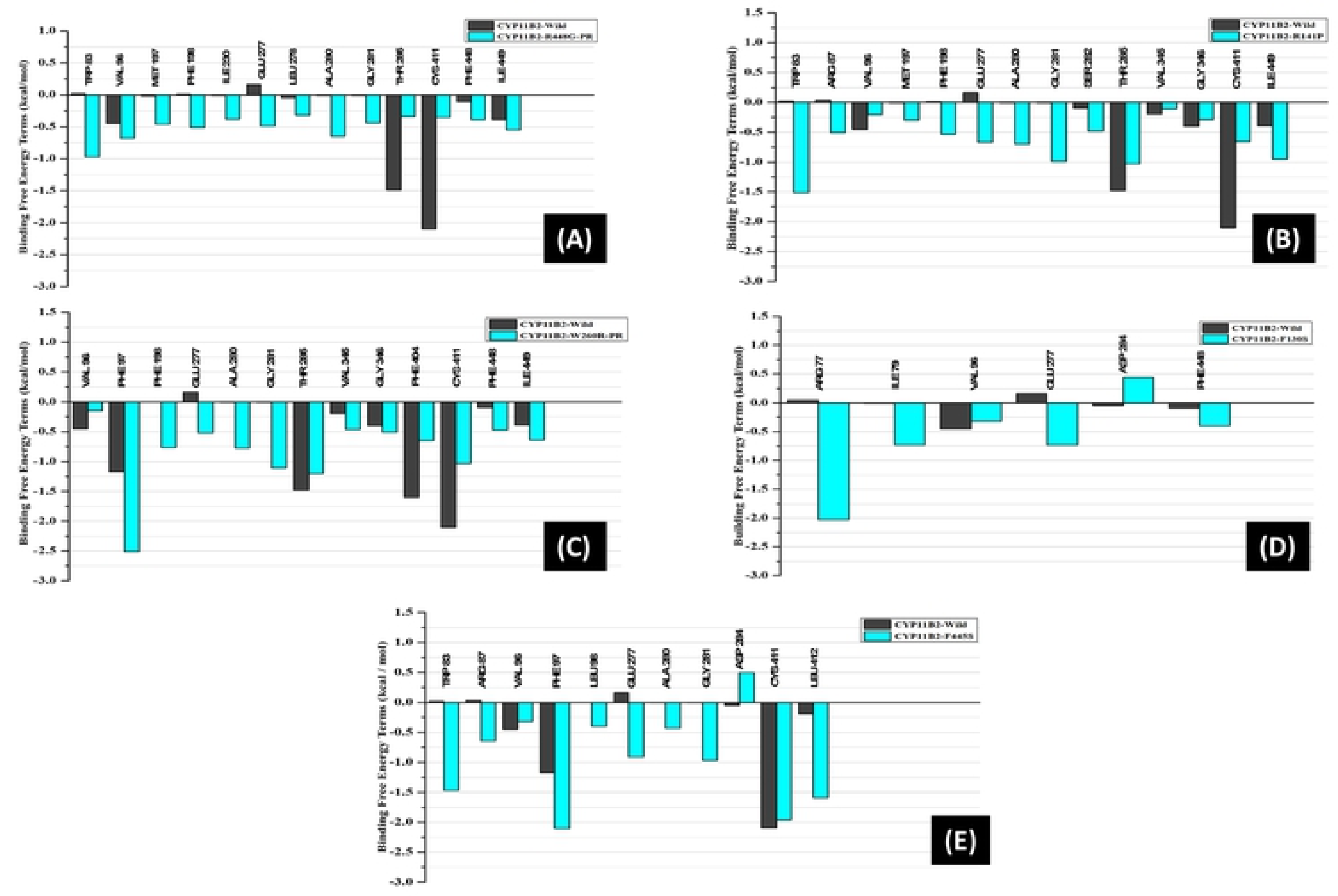

Fig 6 